\title{
An Overview of Smart Shoes in the Internet of Health Things: Gait and Mobility Assessment in Health Promotion and Disease Monitoring
}

\author{
Bjoern M. Eskofier ${ }^{1, *}$ (D), Sunghoon Ivan Lee ${ }^{2}$, Manuela Baron ${ }^{1}$, André Simon ${ }^{1}$, \\ Christine F. Martindale ${ }^{1}$, Heiko Gaßner ${ }^{3}$ and Jochen Klucken ${ }^{3}$ \\ 1 Machine Learning and Data Analytics Lab, Department of Computer Science, \\ Friedrich-Alexander-Universität Erlangen-Nürnberg (FAU), Immerwahrstrasse 2a, 91058 Erlangen, \\ Germany; manuela.baron@fau.de (M.B.); andre.simon.lauf@gmx.de (A.S.); \\ christine.f.martindale@fau.de (C.F.M.) \\ 2 College of Information and Computer Sciences, University of Massachusetts, Amherst, MA 01003, USA; \\ silee@cs.umass.edu \\ 3 Department of Molecular Neurology, University Hospital Erlangen, Friedrich-Alexander-Universität \\ Erlangen-Nürnberg (FAU), 91054 Erlangen, Germany; Heiko.Gassner@uk-erlangen.de (H.G.); \\ jochen.Klucken@uk-erlangen.de (J.K.) \\ * Correspondence: bjoern.eskofier@fau.de; Tel.: +49-9131-85-27297
}

Received: 5 August 2017; Accepted: 14 September 2017; Published: 25 September 2017

\begin{abstract}
New smart technologies and the internet of things increasingly play a key role in healthcare and wellness, contributing to the development of novel healthcare concepts. These technologies enable a comprehensive view of an individual's movement and mobility, potentially supporting healthy living as well as complementing medical diagnostics and the monitoring of therapeutic outcomes. This overview article specifically addresses smart shoes, which are becoming one such smart technology within the future internet of health things, since the ability to walk defines large aspects of quality of life in a wide range of health and disease conditions. Smart shoes offer the possibility to support prevention, diagnostic work-up, therapeutic decisions, and individual disease monitoring with a continuous assessment of gait and mobility. This overview article provides the technological as well as medical aspects of smart shoes within this rising area of digital health applications, and is designed especially for the novel reader in this specific field. It also stresses the need for closer interdisciplinary interactions between technological and medical experts to bridge the gap between research and practice. Smart shoes can be envisioned to serve as pervasive wearable computing systems that enable innovative solutions and services for the promotion of healthy living and the transformation of health care.
\end{abstract}

Keywords: smart shoes; digital health; eHealth; mHealth; machine learning; Parkinson's disease; sensor-based gait analysis; telehealth

\section{Introduction}

Technology developments towards novel healthcare concepts pave the way for the rising area of digital health, which, according to the U.S. Food and Drug Administration (FDA), includes categories such as mobile health (mHealth), health information technology, wearable devices, telehealth and telemedicine, and personalized medicine. Especially, new smart wearable technologies and the health information technology provided by the internet of things would enable a comprehensive view of an individual's life. In this context, mobility is of the utmost importance, as it defines quality of life in healthy living and chronic diseases. In numerous neurological, musculoskeletal, 
and cardiovascular disorders, distinct symptoms reduce motor function and/or cardio-pulmonary capacity, and thereby limit the independence and autonomy of individuals. Even though the disease-causing mechanisms and symptomatic patterns are specific to each disorder, impaired mobility is a typical consequence. This fact makes mobility an important surrogate marker for disease severity, progress, and responsiveness to the prescribed therapies, providing opportunities to assist therapeutic decision making.

The continuous, automatic monitoring of sensor-based information on walking ability and mobility is increasingly exploited to support objective assessment for preventative and proactive disease management and diagnostic workup, and to assist with therapeutic decision-making. In this work, we envision to leverage the emerging Internet of Health Things (IoHT) [1] to enable the application of smart devices for continuous, real-time monitoring. Smart shoes are an attractive form of smart devices for the purpose of mobility assessment for three reasons: (i) smart shoes have a predefined, rigid sensor position on the foot, providing accurate and flexible biomechanical analysis; (ii) smart shoes can be used to monitor gait, a highly stereotype movement that enables the automated assessment of functional biomechanics; and (iii) smart shoes enable a non-obtrusive and non-stigmatizing integration of technology, ultimately improving patient acceptance and long-term adherence. We also envision that the sporting goods industry will produce a growing number of sensor-equipped smart shoes that are capable of monitoring fitness and health conditions. The current limitations of this technology, namely restricted usability to patients, limited battery runtime, and especially restriction to only one shoe model due to a limited availability of instrumentation, will be overcome in the future once this mass-market availability is ensured.

Mobility monitoring technologies demand research in sensor-based data acquisition and its subsequent analysis to support objective and clinically relevant gait analysis outside of conventional clinical environments [2]. The mounting popularity of this research topic is reflected by the annually increasing number of related articles as shown in Figure 1. Thus, this paper aims to provide an overview of the important engineering aspects of smart-shoe applications in the field of IoHT. It will also highlight the differences and requirements of the related engineering and medical needs (Figure 2), which are critical components for effective biomedical engineering solutions.
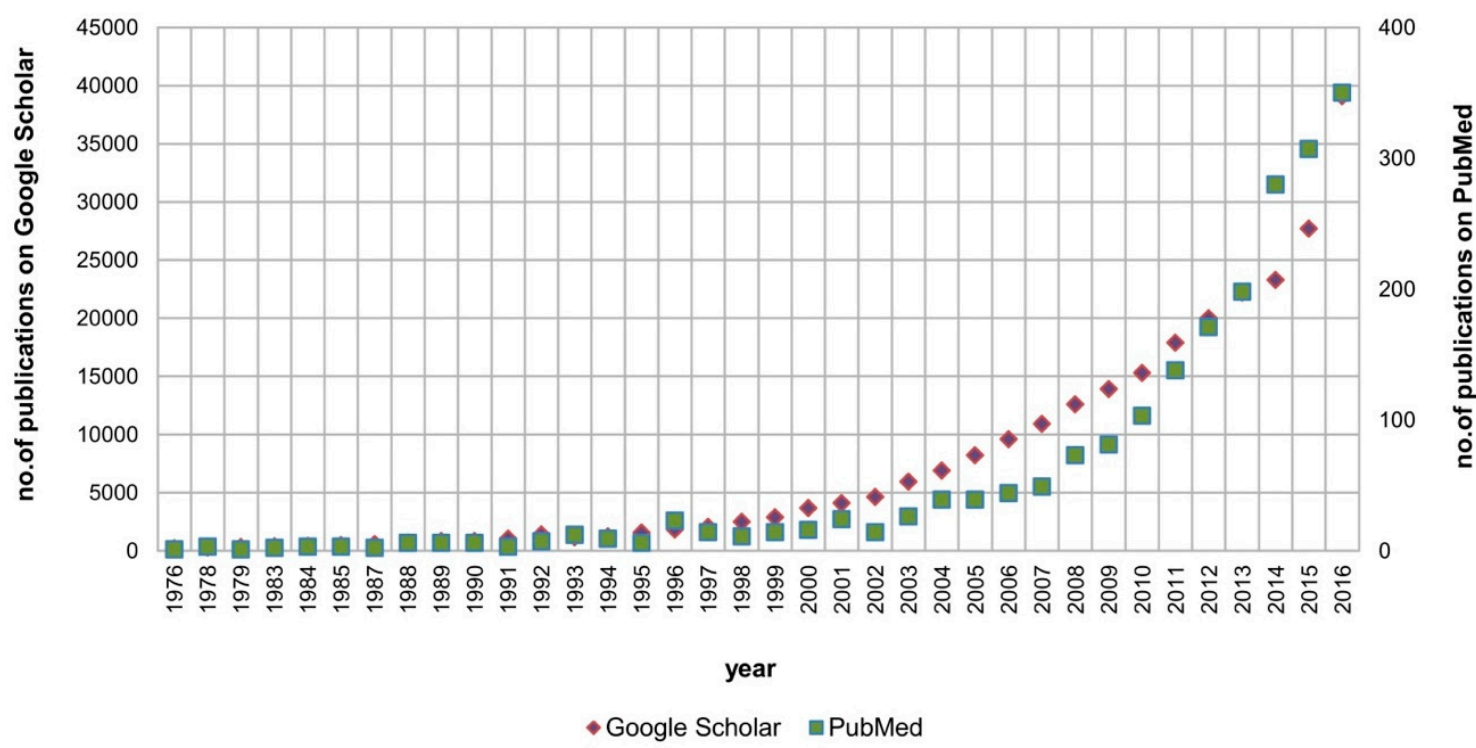

Figure 1. The annual number of publications found using PubMed and Google Scholar on the subject of gait monitoring using smart shoes. The search criteria included "(gait OR shoe OR walking) AND (inertial OR IMU OR sensor OR wearable)"; IMU: Inertial Measurement Unit. 


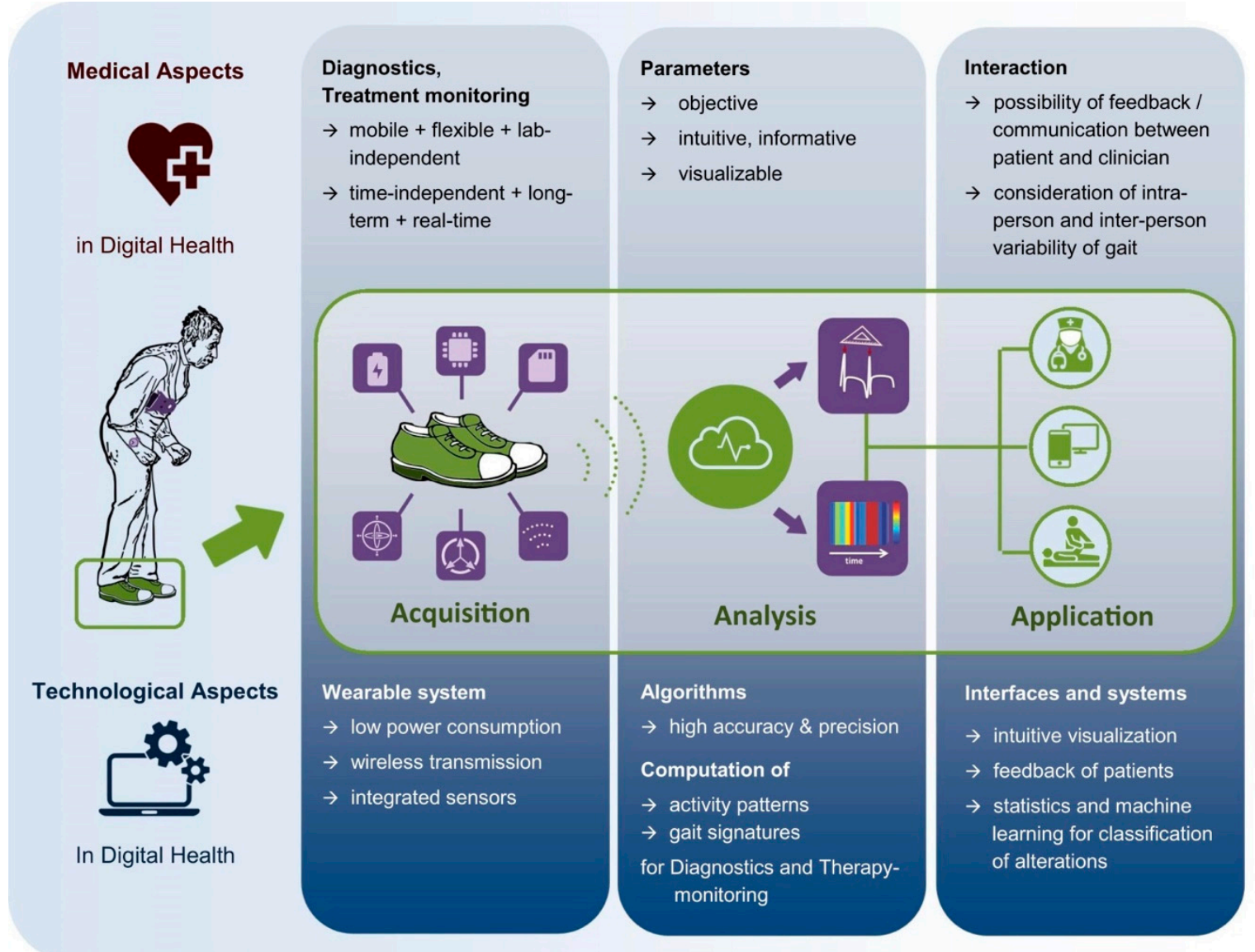

Figure 2. Medical and technological requirements for Internet of Health Things (IoHT) for ambulatory gait monitoring. Icons modified and printed with permission of [3-5]. Patient figure adapted from [6].

\section{Technological Aspects of Smart Shoes for Digital Health}

In this section, we give an overview of the important technological aspects of gait assessment using smart shoes in terms of data acquisition system design, methods for sensor data analysis, and application examples.

\subsection{Acquisition Systems}

Remote gait assessment with smart shoes requires a data acquisition system that will collect sensor data relevant to important gait events while ensuring ease-of-use for long-term compliance. The technical acceptance of the sensing system is particularly important, as the lack of it may lead to insufficient motivation to use the system and reduced data quality. Particularly, an efficient management of power is important to reduce the frequency of required charging of devices, which is closely related to the technical acceptance of the system. Wireless transmission of the recorded data avoids technical manipulation of the device by patients, and simultaneously acts as a data gateway to push the obtained data to the cloud. This area has been intensively studied in the field of Body Sensor Networks [7-9]. In the following two subsections, we will address details regarding the technical acceptance issues, mainly those related to energy consumption and data generation.

\subsubsection{Energy Management Aspects}

Smart shoes assessing gait require optimized energy management to maximize system runtime and minimize technical manipulation by users. Three major aspects influence efficient system 
energy consumption [10]. First, the computational complexity and efficiency of soft- and hardware components need to be optimized. Particularly in the case of wearable systems, energy-aware software implementation methods [11] should be applied, and hardware choices should be made with a focus on low energy consumption [12]. Second, the application context should be taken into account [13]. If the smart shoe recognizes that no data is being produced, the system could go into a low energy consumption (e.g., sleep) mode. Especially for medical monitoring scenarios, continuous and reliable system function must, nevertheless, be ensured [14]. A promising solution for the efficient energy management for smart shoes includes energy harvesting. For instance, energy harvesting from electromagnetic fields [15], other ambient energy sources [16], and human movement have gained considerable research attention, and will be implemented in end-user systems in the foreseeable future [17].

\subsubsection{Data Generation}

Reliable data generation is an important basis for all subsequent analysis and application pipelines. A wide variety of sensors are applicable in the context of smart shoes:

- Relative location and orientation determination using inertial-magnetic measurement units (IMMUs, consisting of an accelerometer, a gyroscope, and a magnetometer) and data fusion algorithms [18]. This data can be used, for example, for gait analysis that is explained later.

- Absolute location determination using satellite navigation systems (GPS, GLONASS, GALILEO) [19], which can also provide time information [20]. Relative and absolute location and orientation determination can be fused using loosely or tightly coupled data fusion algorithms [21]. This data can be used, for example, for wide-range activity tracking in a daily living outdoor context.

- Foot plantar pressure determination using various forms of pressure sensors, which provide information regarding how effectively and efficiently individuals control the distribution of the body weight during gait [22]. This data can be used, for example, for rehabilitation purposes, when a patient should not put too much weight onto a leg after surgery.

- Ambient environmental sensors, such as atmospheric pressure sensors for altitude-dependent activities (e.g., stair climbing or hiking) and local weather information (changes over minutes and hours), and light and sound sensors for context-related information generation.

- Internal status sensors, for example for battery and memory capacity (not discussed further).

Besides sensor data recording, data transmission and storage are also of central importance. The obtained sensor data are typically stored locally on the acquisition system with limited capacity. Therefore, data acquisition systems often have cloud-based data transmission capabilities [23]. For maximum usability, wireless transmission is typically employed, e.g., Bluetooth, Wi-Fi, or proprietary solutions. For our own system, we envision a smart shoe data concentrator that uses wireless battery charging as well as safe and secure cloud data transmission during non-usage periods (Figure 3). It would also contain an offline-data storage module in cases where no cloud data transmission is available or required. Lastly, the data transmission and storage implementation needs to adhere to data safety and security requirements, especially in the case of medical technology systems that manipulate sensitive patient health information.

An overview of sensing technologies with more details (accelerometer, gyroscope, compass sensor, pressure sensors, ... ) can be found in the literature, for example in $[24,25]$. 


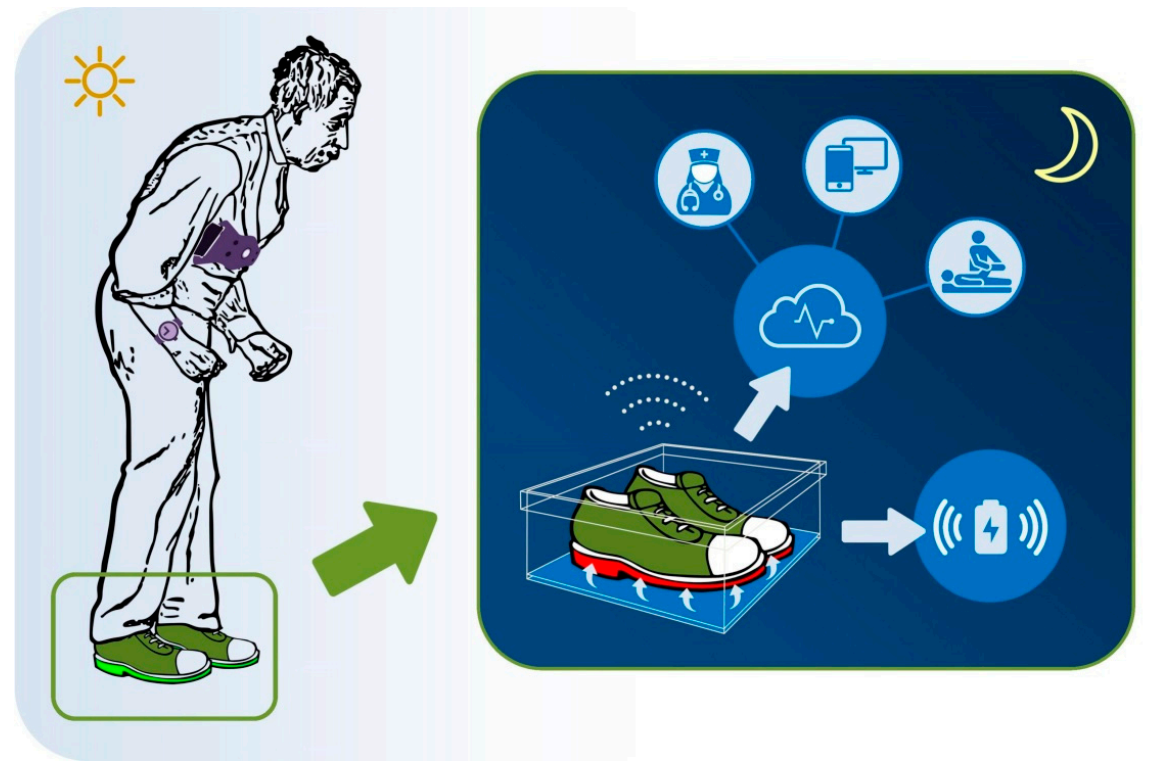

Figure 3. Practical use of smart shoes within the IoHT: capturing gait signals of patients with impaired gait (daytime use), data storage, and battery charging (nighttime use). Icons modified and printed with permission of [3-5]. Patient figure adapted from [6].

\subsection{Analysis Methods}

Once the sensor data are acquired, informative and relevant parameters must be computed to derive the necessary information relevant to important sports, health, and medical events (Figure 4). First, the computed parameters should be able to facilitate an objective assessment of user performance and mobility status. Second, the parameters should be determined with sufficient accuracy and reliability for use in assessment by domain experts. Therefore, the development of analysis methods should be driven by two inherently different concepts, defining: (i) what would be useful to measure from a medical point of view; and (ii) how these measures can be obtained from an engineering point of view. The development of analysis methods is further driven by the application context. For smart shoes specifically, this paper discusses algorithms for two major areas of applications: (1) activity pattern recognition for applications in sports and healthy living; and (2) gait signature derivation for medical diagnostic and treatment contexts.

First, activity patterns (Figure 4, left) provide qualitative information about daily physical activities, such as type, duration, or temporal structure, enabling the assessment of gross changes in the engaged activities or mobility status. Activity pattern computation only requires basic signal processing with no a priori information regarding the subject or the performed activity. Most activity pattern computation can be broadly applied without the need for individualization or for the targeting of specific tasks. Consequently, activity patterns are not suitable for the identification of distinct motions or disease-related gait patterns and characteristics. Figure 4 (left) provides an exemplary illustration of an "activity barcode" that characterizes one's physical activity patterns over time [26].

Second, gait signatures (Figure 4, right) allow the computation of quantitative parameters of specific motions, enabling a more detailed analysis of motion patterns (including variability) [27] and mobility status [28]. Gait signature computation requires tailored signal processing and prior knowledge of the biomechanics of specific movements. It often benefits from a priori knowledge about the subject's biomechanical (anatomical) information and/or the performed activity profiles, e.g., an incorporation of subject-, activity-, or disease-specific information. Nevertheless, this implies a restriction to specific, well-understood disease conditions, which increases the amount of resources required for developing and validating the respective algorithms. As an example, Schülein et al. used a smart shoe gait signature analysis system to objectively measure the gait parameters of geriatric 
patients with and without support of a wheeled walker, aimed at evaluating the individual therapy effect caused by this walking aid [29].

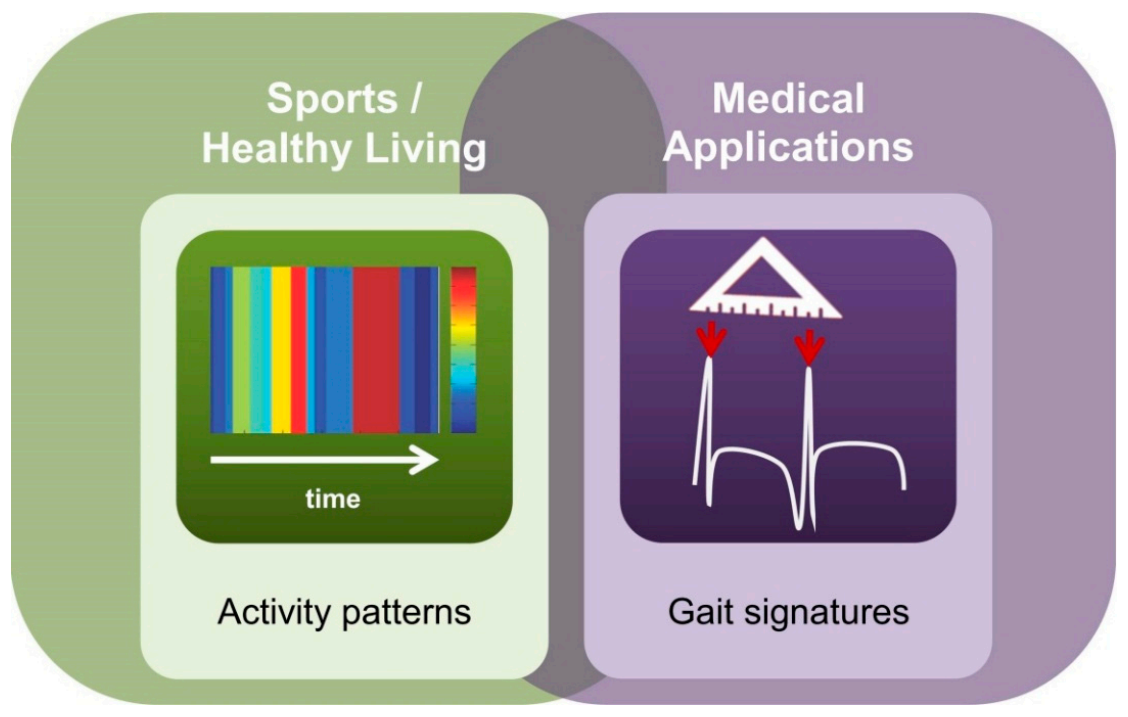

Figure 4. (Left): A schematic illustration of activity pattern variation over time, which is often referred to as an activity barcode [26]; barcode printed with permission of the authors. (Right): assessment of clinically relevant gait signatures [27], e.g., gait cycle length as illustrated in the figure.

In this section, we provide an overview of concrete analysis methods that are relevant for both activity pattern and gait signature computation. As outlined in an excellent review article by [30], there is a common pipeline for inertial sensor-based gait analysis (reproduced and adapted in Figure 5). We will focus on arguably the most important pipeline steps: preprocessing, segmentation, estimation of gait patterns, and recognition of, for example, the clinically applicable mobility status.

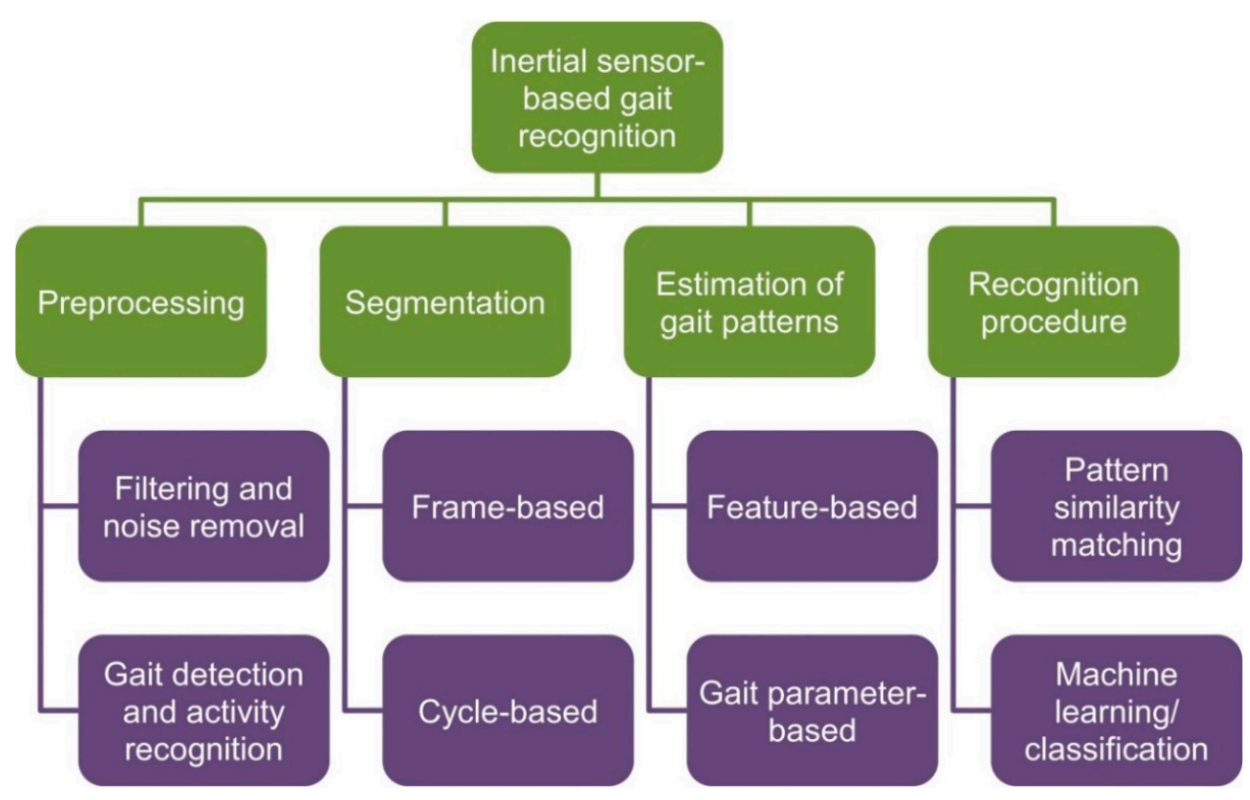

Figure 5. A schematic (hierarchical) representation of a common pipeline for inertial sensor-based gait analysis. Figure modified with permission of the authors of [30]. 


\subsubsection{Preprocessing}

Preprocessing prepares the incoming raw sensor data for subsequent analysis steps. This includes optimizing sampling frequency [31], sensor calibration (e.g., using in-field procedures [32,33]), eliminating irrelevant information from sensor data (e.g., non-human generated sensor noise) using filters [34], drift correction (e.g., using Fourier-based integration [35] or sequential drift correction [23]), and gravity cancellation (e.g., using Kalman filtering [35] or gradient descent-based algorithms [19]). These procedures usually produce gravity-free kinematic information of the sensing unit, such as the estimated orientation and position.

\subsubsection{Segmentation}

Segmentation is important for real-time or ongoing applications where it is impractical or impossible to process the continuously recorded sensor data as a single segment. For activity patterns, these segments are typically fixed-size, moving window-based, and depend on the application context (e.g., minutes for short-term analysis or hours for long-term analysis). For gait signatures, segmentation typically involves two steps: a moving window-based approach in order to detect gait events, allowing for the segmentation of the data into meaningful segments, such as stride or gait bouts. These can be facilitated by simplistic approaches such as peak detection or zero-crossings [36], or sequential model-based approaches such as hidden Markov models [37]. Template-based approaches, such as longest common subsequence [38], Dynamic Time Warping [39], and the multi-dimensional subsequence Dynamic Time Warping approach (msDTW) [40] are also commonly used. The aforementioned methods are often chosen and optimized depending on the application context, as they vary in computational complexity for training, effectiveness, and generality. IMMU data are the most commonly used sensing modality for gait segmentation; however, additional sensors can also be exploited, such as pressure sensors within insoles [41].

\subsubsection{Estimation of Gait Patterns}

When a feature-based machine learning algorithm is employed to recognize important gait events (Section 2.2.4), the extraction of relevant gait parameters (referred to as gait patterns in this work) can significantly reduce the amount of data and help provide an application-specific interpretation of important gait events. Gait patterns can be represented via a finite number of spatiotemporal gait parameters. These parameters can be extracted from the time or frequency domain [42]. Commonly used time domain gait features include local extrema [43,44], adaptive thresholds (or zero) crossings [23,45,46], and gait symmetry indexes [47]. Common frequency domain features include FFT (Fast Fourier Transformation) coefficients [42,48] and wavelet transforms [49]. Other inter-domain features, such as principal component analysis-based approaches [50,51], geometric template matching [52], or curve aligning [53] are also often used.

The aforementioned parameters can be calculated based on a human gait model (e.g., [54]), which divides gait patterns into relevant application-based groups, or on an abstraction model, which directly estimates the gait parameters. Human gait models [27] usually do not involve subject-specific training, but can be problematic in the case of an impaired gait due to their generality. Abstraction models [55] use machine learning methods for a direct estimation of gait patterns. These models are more robust to inter-subject variability, but require substantial data for the training phase [56].

\subsubsection{Recognition of Important Gait Events}

Gait event recognition compares the incoming sensor data, either in the form of preprocessed time-series or computed gait features, to reference characteristics of important gait events in order to enable a qualitative and/or quantitative assessment of mobility status. This is typically conducted either by time-series template matching or feature-based machine learning algorithms [30]. 
For time-series template matching, the computed features are directly compared to fixed patterns or parameters and distance measures, such as a deviation from the subject-specific or disease-specific norm. In [30], several approaches are reported, including Euclidean distance and correlation coefficients [57]. A more sophisticated method is template matching, where Dynamic Time Warping is often adopted [39].

Feature-based machine learning algorithms, in contrast, address the recognition step as a classification problem and are trained a priori by evaluating their classification outcomes. The respective approaches use algorithms such as support vector machines [58], artificial neural networks [59], hidden Markov model classifiers [60], and Gaussian mixture model classifiers [61]. A detailed report on these and further classifiers can be found in a review paper on activity classification [62].

The outcomes of the gait event recognition procedure, i.e., the class or similarity measures, can be used for a variety of applications, including disease classification, progress tracking, evaluation of the effectiveness of therapeutic interventions, and computation of any form of deviation from the norm, either across a population or for individual patients.

\subsection{Application Examples}

In this section, we outline concrete application examples for smart shoe systems that make use of the described acquisition and analysis steps. First, we will outline sports and healthy living applications that benefit from both activity pattern as well as gait event detection algorithms. Then, we focus on medical applications, which are based on detecting specific gait signatures and gait-specific parameters.

\subsubsection{Sports and Healthy Living Applications}

Sports and healthy living applications of smart shoes mainly target the unregulated, consumer-oriented, low-cost lifestyle devices market. Within the so-called "quantified-self" movement, many applications are engaged with activity pattern monitoring. For example, individuals monitor their everyday step count and gait speed [56] with the target to improve habits, or they quantify sports performance with mobile motion capture [63]. Other applications also address gait signature analysis. For example, IMMUs are used to determine joint stress in barefoot running [64], landing patterns [30] and vertical loading [29,30,35,45,65-68], loading while running barefoot [69], the effects of a high-heeled gait [70], the effects of rollover footwear [71], and the influence of the long-term wearing of unstable shoes [67].

\subsubsection{Medical Applications}

Medical applications target the heavily regulated, patient-oriented, high-accuracy medical devices market. Most applications that are based on smart shoe technologies aim for impairment-specific gait signature assessment. A recent review article [25] gives an excellent overview regarding the applications of smart shoes in the medical domain, including fantastic overview tables that compare related work with specific items. In their study, the authors found that $29 \%$ of the included articles address Parkinson's disease, 17\% Cerebral Palsy, and 13\% orthoses, as summarized in Figure 6.

In [28], Klucken et al. integrated clinical gait assessment in a smart shoe for the machine learning-based discrimination of healthy controls and patients in increasing stages of Parkinson's disease (PD). The results proved that discrimination using these objective measurements is feasible.

PD has also been in the focus of different review articles [72,73] discussing the applicability and possibilities of especially wearable monitoring technologies. A reason for this is that PD is an excellent model disease for the development of mHealth technologies [74], where the results will be generalizable to a variety of other neurological and musculoskeletal conditions [75]. Wearables' application to PD was also investigated in an excellent article by Del Din and colleagues [76]. Caudron et al. introduced a real-time system that could provide visual feedback to improve postural stability in PD [77]. Feedback was given in the form of depicting the geometry of the patient's body. The authors recruited 17 patients with $\mathrm{PD}$, and tested their balance after pull-tests under various conditions, e.g., eyes closed/open with or without visual biofeedback. 


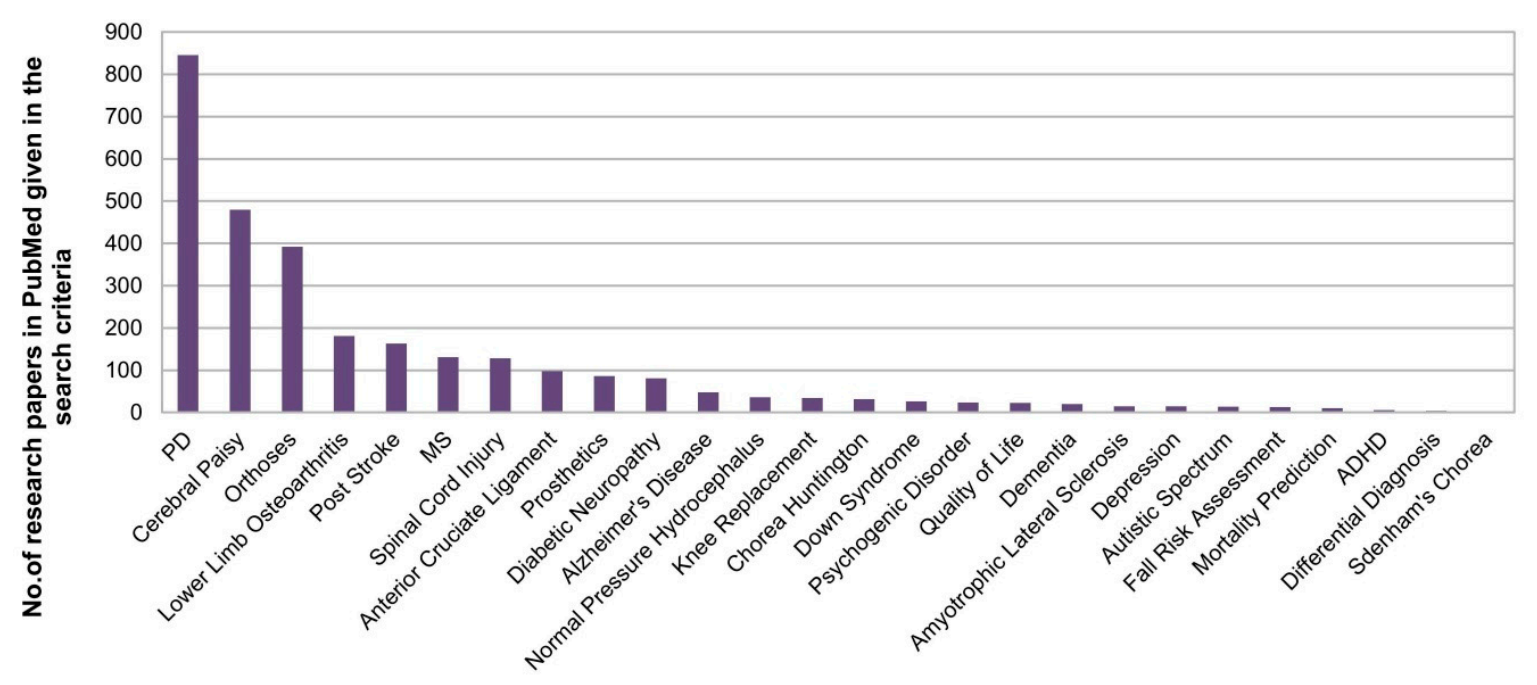

Gait-impairing diseases researched using gait analysis

Figure 6. Distribution of research efforts on different gait pathologies (data originates from research papers published between 1970 and 2016). Figure modified with permission of the authors of [25]. PD: Parkinson's disease; MS: multiple sclerosis; ADHD: attention deficit hyperactivity disorder.

A comparable study by Horak et al. examined the influence of smart shoes on stability and gait rehabilitation [78]. The authors highlighted the potential to individualize the diagnosis of gait impairment and the adaptation of treatment to the specific difficulties of each subject. According to the authors, it is crucial that therapists recognize the benefits and limitations of technology-enriched therapy. They also advocated that further steps towards telerehabilitation and digital health would be beneficial.

In [79], Schwenk et al. targeted the discrimination of frailty status in geriatric patients, i.e., non-frail, pre-frail, and frail. The study was based on data collected for the Arizona frailty cohort, and employed a wearable sensor-based at-home assessment of gait, balance, and physical activity. Their results showed that stride length and double support time were the most sensitive parameters for discriminating the three conditions.

Lee et al. validated the use of smart shoes during a 10-m walking test to quantify the functional level in individuals with spinal cord disorders [80] and to predict their postoperative outcomes [81]. Their results support the view that the measurement of spatiotemporal gait characteristics from smart shoes can provide an accurate representation of functional level and predictions of surgical outcomes.

A different application was addressed by Perrier et al. in [82]. The authors focused on diabetics at risk of suffering foot ulcers. The authors designed a wireless, customizable, and washable smart sock with pressure-sensitive fibers that computed stress indicators based on pressure data. A subject-specific finite element foot model was introduced to provide real-time feedback to help patients prevent foot ulcers.

A further aspect that has been researched is glaucoma patients. Ma and colleagues [83] investigated gait function in this patient population, and presented a wearable wireless sensor system designed for real-time gait pattern analysis in glaucoma patients. The system was utilized in a randomized clinical trial with 19 participants, both glaucoma patients and age-matched healthy controls. Their results demonstrated that machine learning algorithms achieve an accuracy of over $80 \%$ in distinguishing extracted gait features of those with glaucoma from healthy individuals.

These findings show that smart shoe technology has great potential to allow accurate diagnostics of gait impairments and their progression during the rehabilitation process. Information obtained from smart shoes could be leveraged to enable individually-tailored treatment plans, and hence optimize clinical outcomes. Studies also suggest that applications need to be carefully designed to reflect gait 
characteristics that are specific to the target medical conditions, which will be discussed in detail in the following section.

\section{Medical Aspects of Smart Shoes for Digital Health}

Gait impairment is a common symptom in several diseases, and affects activities of daily living and quality of life $[65,68,84]$. Among others, diseases with typical gait impairments are PD, multiple sclerosis (MS), cerebral palsy (CP), osteoarthritis, rheumatism, spinal cord disorders, and sarkopenia. Quantitative gait assessment of activity patterns has the potential to support clinical diagnostics and therapeutic decisions [85], compensating for the fact that clinical motor assessments are subjective in that they vary between investigators and depend on the experience of clinicians [86]. Sensor-based, mobile gait systems are able to provide objective, spatiotemporal gait parameters recorded in a flexible environment without being limited to motion labs, such as those measures found using gait analysis carpets or high-precision three-dimensional (3D)-motion capture systems. Wearable sensors have been shown to provide congruent results in patient cohorts with good accuracy and precision compared to gold-standard laboratory systems $[27,87]$.

As an example for typical gait impairment, patients suffering from PD show four cardinal symptoms: bradykinesia, rigor, tremor, and postural instability [88]. These major symptoms, with the exception of tremor, affect gait and consequently PD disease characteristics are associated with gait impairment. The clinical gold standard in rating motor symptoms is the Unified Parkinson Disease Rating Scale Part III (UPDRS-III) as described from the Movement Disorder Society [89]. However, since only 2 out of 16 items of the UPDRS-III assess walking tasks, gait is under-represented in this clinical score, and complementary measures are necessary. Sensor-based gait analysis systems are able to close this gap (Figure 7).
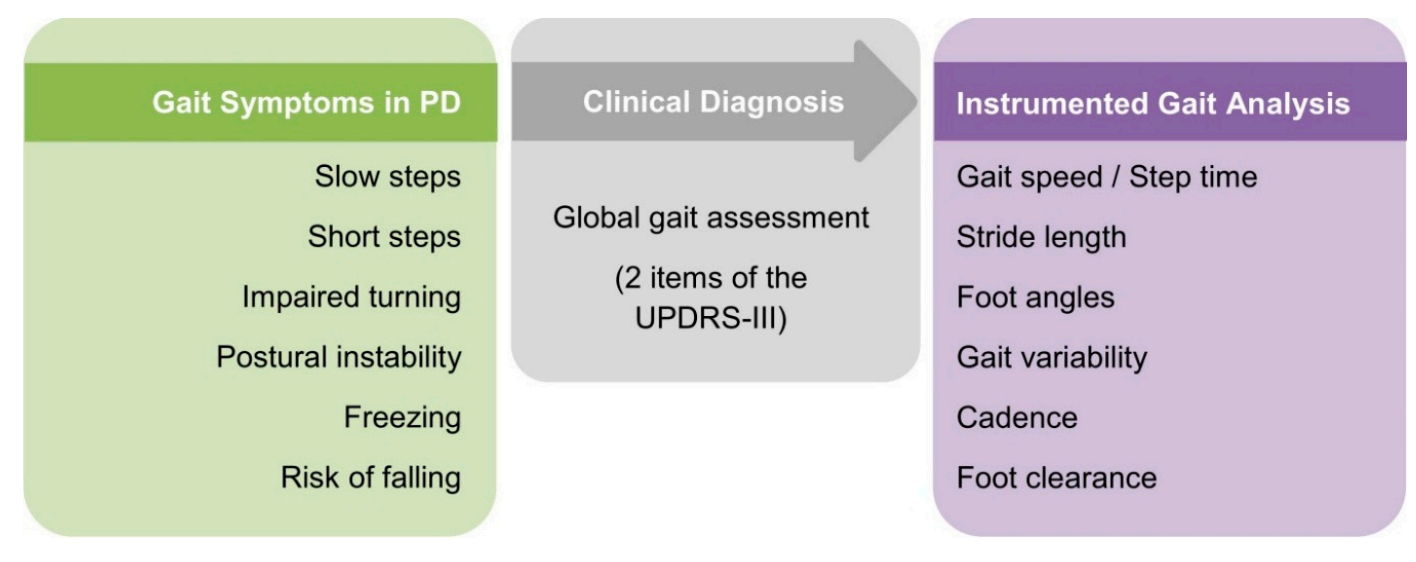

Figure 7. Clinical diagnosis based on the Unified Parkinson Disease Rating Scale, part III motor score (UPDRS-III) is not able to adequately reflect all gait impairments that occur in Parkinson's disease (PD). An instrumented gait analysis complements clinical assessment by providing different objective parameters evaluating the broad spectrum of gait disturbances in PD.

Gait and mobility are strongly correlated with quality of life (QoL) in PD, as summarized in several reviews $[66,90,91]$. Objective gait assessment supports not only the clinical diagnostic workup in PD, but also provides monitoring measures in the course of the disease to reflect the severity of motor impairment and thus QoL.

With disease progression, PD patients develop motor fluctuations during the day, and thus depend on timely medication intake and monitoring of the therapeutic effects on their mobility [74]. Home monitoring approaches could cover this range of gait mobility over the course of the day, and serve as complementary technology with the aim to optimize multidisciplinary medical care concepts for PD patients. For this purpose, the measured physical and physiological data need to go 
beyond pure smart shoe applications, and other biosignals (speech, upper extremity, sleep, ... ) need to be investigated. This has, for example, been extensively discussed in [92].

In a pilot study, a telemedical application that allows PD patients to attend clinical appointments via video conference at home has been evaluated with 34 PD patients using a patient satisfaction questionnaire. PD patients' self-reporting revealed that the average costs related to the travel to the doctor could be reduced by $\$ 200$, and commute time by $209 \mathrm{~min}$ [93]. This supports that telemedicine is a cost- and time-effective approach. Another group developed an instrumented Timed Up and Go test (iTUG) supported by wearable sensors to receive complementary information by analyzing gait characteristics in different test phases (i.e., sit-to-stand, walking, turning, and turn-to-sit) instead of just interpreting the time patients take to complete the test $[94,95]$. The iTUG was assessed in PD subjects and controls in a laboratory as well as in a home environment. iTUG measurement at home has been shown to be feasible, providing comparable results in differentiating early PD patients and controls using sensor-based parameters such as cadence, angular velocity of arm-swing, turning time, and turn-to-sit time. In a therapy monitoring study observing how walking aids may affect risk of falling related gait parameters in 106 geriatric patients, wearable sensors have recently been shown to objectively evaluate the positive impact of a wheeled walker on gait stability [29].

Despite promising benefits in the provision of complementary information for clinicians and the delivery of high-quality, efficient care, sensor-based objective measures are at present not implemented into complex healthcare concepts and also not refunded by healthcare providers [74,92].

Indeed, there are challenges with analyzing gait in complex diseases such as PD, where the spectrum of symptoms affecting individual quality of life range from diverse motor-symptoms, including gait impairment, to non-motor symptoms [91,96]. Thus, mobility limiting gait patterns are heterogeneous even in PD patients in the same disease stage. In addition, if motor-fluctuations appear, they can vary throughout the course of the day. The most advanced applications to monitor motor-fluctuations using wrist-worn inertial sensors show the limits of activity patterns in enabling the timely, exact prediction of the motor state of a patient [86]. Therefore, complementary to wrist-worn sensor-based activity patterns, gait signatures could help to identify and resolve individual motor patterns before enabling a validated quantification of individualized gait and motor impairments. Hence, sensor-based measures have the potential to support clinical trials and patient care in the hospital or home environment by continuous monitoring of clinically relevant targets, including motor-fluctuations and falls.

\section{Discussion and Conclusions}

Although wearable sensors are ubiquitously present on the market for health and sport activities, the same is not true for the medical application sector. Usable and useful patient-empowering telemedical applications in the disease context are still mainly research examples. This is, in part, due to the substantially higher requirements regarding development and regulatory hurdles for medical products. Nevertheless, many systems are on the verge of entering the new area of digital medicine. They have great potential to increase quality of life and care, prevent comorbidity, and improve healthcare processes. Wearable sensors will change the way that healthcare is conducted. The associated economic changes that this process will bring, as well as the requirements for the regulation of data and ethics, are largely unaddressed at this point in time.

In any case, smart shoes as part of the IoHT will play an important role in this change process. Gait is an ideal target parameter, since it defines mobility and autonomy as the major aspects of quality of life in health and disease. A review article by Muro-de-la-Herran et al. [97] underlines this, reporting a shift of interest towards body-worn sensor-based systems for gait analysis. In our opinion, the field of gait analysis should move even more from instrumented tests that increase the objectiveness and transparency of classical healthcare strategies towards pervasive telemedicine solutions that enable new monitoring concepts for continuous and individualized healthcare integrated into the everyday environment of patients. 
However, in order to move the medical application field forward, there is a definite need for more translational research between engineering and medical experts. According to a recently published review article in the field of gait assessment using inertial sensors [25], only 30\% of the 669 analyzed papers include relevant patient cohorts, and only $13 \%$ of the papers actually report on an application in an operational clinical environment required for the highest technology readiness levels.

In our opinion, the steps required to pave the way for a better clinical application of wearable sensors are the following:

(1) address disease-specific gait characteristics by objective and quantifiable gait parameters

(2) assess the validity and reproducibility of gait-related measures

(3) provide secure, safe, and reliable telemedical communication platforms linking wearable sensor-based diagnostics, patient, therapists, and care givers

(4) integrate the medical and technological requirements during the development phase of wearable sensors

(5) target user experience, both from medical- and consumer-use perspectives; this should also be a target of academic investigations using statistics and discussion on user compliance for different types of smart shoes (materials, features, usability)

(6) include regulatory and economic requirements in the development process

(7) motivate researchers to comprehensively address both technological solutions and medical requirements, and demonstrate their relevance in operational clinical environments.

The example of sensor-based gait assessment nicely reveals that even though activity patterns are suitable to generate relevant parameters for healthy living, more advanced algorithms and experimental designs are needed for sport applications, but are needed even more importantly in the disease context. Close collaboration between engineers and clinicians is necessary to understand the needs of both fields (technical and medical), and to finally develop a practical solution that fits with the technical requirements and that is able to support medical experts in clinical diagnostics and care. As a third group, it is necessary to include patients in this digital health system, since the aim is to improve their care concept. In this context, it is necessary to evaluate the patients' needs and satisfaction during the use of newly developed telemedical applications. The more patients realize that this telehealth system supports their therapy or quality of life, the more interesting it is for affected people.

Acknowledgments: Bjoern M. Eskofier gratefully acknowledges the support of the German Research Foundation (DFG) within the framework of the Heisenberg professorship program (grant number ES 434/8-1), and furthermore from grants from adidas AG and Bosch $\mathrm{GmbH}$. Jochen Klucken reports grants and personal fees from Astrum IT GmbH, Teva GmbH, Licher MT GmbH, UCB Pharma GmbH, Ever Pharma GmbH, Desitin Arzneimittel $\mathrm{GmbH}$, Abbvie $\mathrm{GmbH} \& \mathrm{Co}$. KG, Biogen GmbH, and GlaxoSmithKline GmbH \& Co. KG. All authors gratefully acknowledge funding from Emerging Fields Initiative (EFI), FAU Erlangen-Nuernberg, during the preparation of this manuscript. The other authors (Manuela Baron, André Simon, Christine F. Martindale, Heiko Gaßner) report no conflict of interest.

Author Contributions: All authors (Bjoern M. Eskofier, Sunghoon Ivan Lee, Manuela Baron, André Simon, Christine F. Martindale, Heiko Gaßner, Jochen Klucken) have made substantial contributions to the following: (1) drafting the article or revising it critically for important intellectual content, (2) final approval of the version to be submitted. Each of the authors has read and concurs with the content in the manuscript. The manuscript and the material within have not been and will not be submitted for publication elsewhere.

Conflicts of Interest: Bjoern M. Eskofier and Jochen Klucken each have a 24.5\% equity in Portabiles HealthCare Technologies $\mathrm{GmbH}$, and furthermore have a patent related to gait assessment pending (EP 16174268.9). The founding sponsors named above had no role in the design of the paper; in the collection, analyses, or interpretation of data; in the writing of the manuscript, and in the decision to publish the paper.

\section{References}

1. Pasluosta, C.F.; Gaßner, H.; Winkler, J.; Klucken, J.; Eskofier, B.M. An emerging era in the management of parkinson's disease: Wearable technologies and the internet of things. Biomed. Health Inform. 2015, 19, 9. 
2. Lord, S.; Galna, B.; Rochester, L. Moving forward on gait measurement: Toward a more refined approach. Mov. Disord. 2013, 28, 1534-1543. [CrossRef] [PubMed]

3. Freeiconspng. Available online: http:/ / freeiconspng.com/ (accessed on 24 March 2017).

4. Flaticon. Available online: https:/ /www.flaticon.com/ (accessed on 24 March 2017).

5. The Noun Project. Available online: https://thenounproject.com/ (accessed on 24 March 2017).

6. Parkinson, J. An Essay on the Shaking Palsy; Sherwood, Neely, Jones: London, UK, 1817.

7. Chen, M.; Gonzalez, S.; Vasilakos, A.; Cao, H.; Leung, V.C.M. Body area networks: A survey. Mob. Netw. Appl. 2011, 16, 171-193. [CrossRef]

8. Fortino, G.; Giannantonio, R.; Gravina, R.; Kuryloski, P.; Jafari, R. Enabling effective programming and flexible management of efficient body sensor network applications. IEEE Trans. Hum.-Mach. Syst. 2013, 43, 115-133. [CrossRef]

9. Raveendranathan, N.; Galzarano, S.; Loseu, V.; Gravina, R.; Giannantonio, R.; Sgroi, M.; Jafari, R.; Fortino, G. From modeling to implementation of virtual sensors in body sensor networks. IEEE Sens. J. 2012, 12, 583-593. [CrossRef]

10. Meckler, S.; Goridko, V.; Ylli, K.; Hoffmann, D.; Willmann, A.; Hehn, T.; Schwaab, M.; Ehrbrecht, B.; Manoli, Y. Pedestrian localization based on a shoe-integrated energy autarkic mobile cyber-physical system. In Proceedings of the VDE-Kongress, Mannheim, Germany, 7-8 November 2016; pp. 1-6.

11. Jensen, U.; Kugler, P.; Ring, M.; Eskofier, B.M. Approaching the accuracy-cost conflict in embedded classification system design. Pattern Anal. Appl. 2016, 3, 839-855. [CrossRef]

12. Ochoa, C.E.; Aries, M.B.C.; van Loenen, E.J.; Hensen, J.L.M. Considerations on design optimization criteria for windows providing low energy consumption and high visual comfort. Appl. Energy 2012, 95, 238-245. [CrossRef]

13. Park, W.K.; Choi, C.S.; Lee, I.W.; Jang, J. Energy efficient multi-function home gateway in always-on home environment. IEEE Trans. Consum. Electron. 2010, 56, 106-111. [CrossRef]

14. Pantelopoulos, A.; Bourbakis, N.G. Prognosis-Awearable health-monitoring system for people at risk: Methodology and modeling. IEEE Trans. Inform. Technol. Biomed. 2010, 14, 613-621. [CrossRef] [PubMed]

15. Georgiou, O.; Mimis, K.; Halls, D.; Thompson, W.; Gibbins, D. How many wi-fi aps does it take to light a lightbulb? IEEE Access 2016, 4, 3732-3746. [CrossRef]

16. Tan, Y.K.; Panda, S.K. Energy harvesting from hybrid indoor ambient light and thermal energy sources for enhanced performance ofwireless sensor nodes. IEEE Trans. Ind. Electron. 2011, 58, 4424-4435. [CrossRef]

17. Elouze, N.; Rekhis, S.; Boudriga, N. Forensic investigation of digital crimes in healthcare applications. In Data Mining Trends and Applications in Criminal Science and Investigations; IGI Global: Hershey, PA, USA, 2016.

18. Bergamini, E.; Ligorio, G.; Summa, A.; Vannozzi, G.; Cappozzo, A.; Sabatini, A.M. Estimating orientation using magnetic and inertial sensors and different sensor fusion approaches: Accuracy assessment in manual and locomotion tasks. Sensors 2014, 14, 18625-18649. [CrossRef] [PubMed]

19. Madgwick, S.O.H.; Harrison, A.J.L.; Vaidyanathan, R. Estimation of imu and marg orientation using a gradient descent algorithm. In Proceedings of the IEEE International Conference on Rehabilitation Robotics, Zurich, Switzerland, 29 June-1 July 2011; pp. 1-7.

20. Mahony, R.; Hamel, T.; Pflimlin, J.M. Nonlinear complementary filters on the special orthogonal group. IEEE Trans. Autom. Control 2008, 53, 1203-1217. [CrossRef]

21. Daniec, K.; Jędrasiak, K.; Koteras, R.; Nawrat, A. Embedded micro inertial navigation system. Appl. Mech. Mater. 2013, 249-250, 1234-1246. [CrossRef]

22. Noshadi, H.; Dabiri, F.; Ahmadian, S.; Amini, N.; Sarrafzadeh, M. Hermes: Mobile system for instability analysis and balance assessment. ACM Trans. Embed. Comput. Syst. 2013, 12, 1-24.

23. Mariani, B.; Hoskovec, C.; Rochat, S.; Buela, C.; Penders, J.; Aminian, K. 3d gait assessment in young and elderly subjects using foot-worn inertial sensors. J. Biomech. 2010, 43, 2999-3006. [CrossRef] [PubMed]

24. Wang, Z.; Yang, Z.; Dong, T. A review of wearable technologies for elderly care that can accurately track indoor position, recognize physical activities and monitor vital signs in real time. Sensors 2017, 17, 341. [CrossRef] [PubMed]

25. Chen, S.; Lach, J.; Lo, B.; Yang, G.Z. Toward pervasive gait analysis with wearable sensors: A systematic review. Biomed. Health Inform. 2016, 20, 1521-1537. [CrossRef] [PubMed]

26. Paraschiv-Ionescu, A.; Perruchoud, C.; Buchser, E.; Aminian, K. Barcoding human physical activity to assess chronic pain conditions. PLoS ONE 2012, 7, 12. [CrossRef] [PubMed] 
27. Rampp, A.; Barth, J.; Schuelein, S.; Gaßmann, K.G.; Klucken, J.; Eskofier, B. Inertial sensor-based stride parameter calculation from gait sequences in geriatric patients. IEEE Trans. Biomed. Eng. 2015, 62, 1089-1097. [CrossRef] [PubMed]

28. Klucken, J.; Barth, J.; Kugler, P.; Schlachetzki, J.; Henze, T.; Marxreiter, F.; Kohl, Z.; Steidl, R.; Hornegger, J.; Eskofier, B.; et al. Unbiased and mobile gait analysis detects motor impairment in parkinson's disease. PLoS ONE 2013, 8, 1-9. [CrossRef] [PubMed]

29. Schulein, S.; Barth, J.; Rampp, A.; Rupprecht, R.; Eskofier, B.M.; Winkler, J.; Gassmann, K.G.; Klucken, J. Instrumented gait analysis: A measure of gait improvement by a wheeled walker in hospitalized geriatric patients. J. Neuroeng. Rehabil. 2017, 14, 18. [CrossRef] [PubMed]

30. Sprager, S.; Juric, M.B. Inertial sensor-based gait recognition: A review. Sensors 2015, 15, 39. [CrossRef] [PubMed]

31. Brønd, J.C.; Arvidsson, D. Sampling frequency affects the processing of actigraph raw acceleration data to activity counts. J. Appl. Physiol. 2016, 120, 362-369. [CrossRef] [PubMed]

32. Bastian, T.; Maire, A.; Dugas, J.; Ataya, A.; Villars, C.; Gris, F.; Perrin, E.; Caritu, Y.; Doron, M.; Blanc, S.; et al. Automatic identification of physical activity types and sedentary behaviors from 3-axial accelerometer: Lab-based calibrations are not enough. J. Appl. Physiol. 2015, 118, 716-722. [CrossRef] [PubMed]

33. Ferraris, F.; Grimaldi, U.; Parvis, M. Procedure for effortless in-field calibration of three-axis rate gyros and accelerometers. Sens. Mater. 1995, 7, 311-330.

34. Tong, K.; Granat, M.H. A practical gait analysis system using gyroscopes. Med. Eng. Phys. 1999, $21,87-94$. [CrossRef]

35. Sabatini, A.M.; Ligorio, G.; Mannini, A. Fourier-based integration of quasi-periodic gait accelerations for drift-free displacement estimation using inertial sensors. BioMed. Eng. OnLine 2015, 14, 1-18. [CrossRef] [PubMed]

36. Sabatini, A.M.; Martelloni, C.; Scapellato, S.; Cavallo, F. Assessment of walking features from foot inertial sensing. IEEE Trans. Biomed. Eng. 2005, 52, 9. [CrossRef] [PubMed]

37. Mannini, A.; Sabatini, A.M. Gait phase detection and discrimination between walking-jogging activities using hidden markov models applied to foot motion data from a gyroscope. Gait Posture 2012, 36, 657-661. [CrossRef] [PubMed]

38. Jarchi, D.; Wong, C.; Kwasnicki, R.M.; Heller, B.; Tew, G.A.; Yang, G.Z. Gait parameter estimation from a miniaturized ear-worn sensor using singular spectrum analysis and longest common subsequence. IEEE Trans. Biomed. Eng. 2014, 61, 1261-1273. [CrossRef] [PubMed]

39. Barth, J.; Oberndorfer, C.; Pasluosta, C.; Schuelein, S.; Gaßner, H.; Reinfelder, S.; Kugler, P.; Schuldhaus, D.; Winkler, J.; Klucken, J.; et al. Stride segmentation during free walk movements using multi-dimensional subsequence dynamic time warping on inertial sensor data. Sensors 2015, 15, 22. [CrossRef] [PubMed]

40. Ten Holt, G.A.; Reinders, M.J.T.; Hendriks, E.A. Multi-dimensional dynamic time warping for gesture recognition. In Proceedings of the Thirteenth Annual Conference of the Advanced School for Computing and Imaging, Heijen, The Netherlands, 13-15 June 2007; pp. 1-8.

41. González, I.; Fontecha, J.; Hervás, R.; Bravo, J. An ambulatory system for gait monitoring based on wireless sensorized insoles. Sensors 2015, 15, 16589-16613. [CrossRef] [PubMed]

42. Sejdic, E.; Lowry, K.A.; Bellanca, J. A comprehensive assessment of gait accelerometry signals in time, frequency and time-frequency domains. J. Neural Syst. 2014, 22, 603-612. [CrossRef] [PubMed]

43. Trojaniello, D.; Cereatti, A.; Della Croce, U. Accuracy, sensitivity and robustness of five different methods for the estimation of gait temporal parameters using a single inertial sensor mounted on the lower trunk. Gait Posture 2014, 40, 487-492. [CrossRef] [PubMed]

44. Arminian, K. Temporal feature estimation during walking using miniature accelerometers: An analysis of gait improvement after hip arthroplasty. Med. Biol. Eng. Comput. 1999, 37, 686-691. [CrossRef]

45. Shin, S.H.; Park, C.G. Adaptive step length estimation algorithm using optimal parameters and movement status awareness. Med. Eng. Phys. 2011, 33, 1064-1071. [CrossRef] [PubMed]

46. Tadano, S.; Takeda, R.; Tohyama, H. Gait characterization for osteoarthritis patients using wearable gait sensors (h-gait systems). J. Biomech. 2016, 49, 684-690. [CrossRef] [PubMed]

47. Sant'Anna, A.; Wickstroem, N.; Eklund, H.; Zuegner, R. Assessment of gait symmetry and gait normality using intertial sensors: In-lab and in-situ evaluation. In Communications in Computer and Information Science; Springer: Berlin/Heidelberg, Germany, 2012; pp. 239-254. 
48. Mazilu, S.; Blanke, U.; Hardegger, M.; Troester, G.; Gazit, E.; Dorfman, M.; Hausdorff, J.M. Gaitassist: A wearable assistant for gait training and rehabilitation in parkinson's disease. In Proceedings of the IEEE International Conference on Pervasive Computing and Communications Demonstrations, Budapest, Hungary, 24-28 March 2014; pp. 135-137.

49. McCamley, J.; Donati, M.; Grimpampi, E.; Mazzà, C. An enhanced estimate of reinitial contact and final contact instants of time using lower trunk inertial sensor data. Gait Posture 2012, 36, 316-318. [CrossRef] [PubMed]

50. Federolf, P.A.; Boyer, K.A.; Andriacchi, T.P. Application of principal component analysis in clinical gait research: Identification of systematic differences between healthy and medical knee-osteoarthritic gait. J. Biomech. 2013, 46, 2173-2178. [CrossRef] [PubMed]

51. Sprager, S. Gait identification using cumulants of accelerometer data. In Proceedings of the Sensors, Signals, Visualization, Imaging, Simulation and Materials, Baltimore, MD, USA, 7-9 November 2009; pp. 94-99.

52. Frank, J.; Mannor, S.; Pineau, J. Time series anaylsis using geometric template matching. Trans. pattern Anal. Mach. Intell. 2013, 35, 740-754. [CrossRef] [PubMed]

53. Sun, H.; Yuao, T. Curve aligning approach for gait authentication based on a wearabe accelerometer. Physiol. Meas. 2012, 33, 1111-1120. [CrossRef] [PubMed]

54. Lord, S.; Galna, B.; Verghese, J.; Coleman, S.; Burn, D.; Rochester, L. Independent domains of gait in older adults and associated motor and nonmotor attributes: Validation of a factor analysis approach. J. Gerontol. A Biol. Sci. Med. Sci. 2013, 68, 820-827. [CrossRef] [PubMed]

55. Hannink, J.; Kautz, T.; Pasluosta, C.; Gassmann, K.G.; Klucken, J.; Eskofier, B.M. Sensor-based gait parameter extraction with deep convolutional neural networks. IEEE J. Biomed. Health Inform. 2016, 21, 85-93. [CrossRef] [PubMed]

56. Yang, S.; Li, Q. Inertial sensor-based methods in walking speed estimation: A systematic review. Sensors 2012, 12, 6102-6116. [CrossRef] [PubMed]

57. Ngo, T.T.; Makihara, Y.; Nagahara, H.; Mukaigawa, Y.; Yagi, Y. The largest inertial sensor-based gait database and performance evaluation of gait-based personal authentication. Pattern Recognit. 2014, 47, $228-237$. [CrossRef]

58. Mannini, A.; Trojaniello, D.; Cereatti, A.; Sabatini, A.M. A machine learning framework for gait classification using inertial sensors: Application to elderly, post-stroke and huntington's disease patients. Sensors 2016, 16, 134. [CrossRef] [PubMed]

59. Nukala, B.T.; Shibuya, N.; Rodriguez, A.; Tsay, J. An efficient and robust fall detection system using wireless gait analysis sensor with artificial neural network (ann) and support vector machine (svm). Open J. Appl. Biosens. 2013, 3, 29-39. [CrossRef]

60. Nickel, C.; Busch, C.; Rangarajan, S. Using hidden markov models for accelerometer-based biometric gait recognition. In Proceedings of the IEEE 7th International Colloquium on Signal Processing and Its Applications, Penang, Malaysia, 4-6 March 2011; Volume 7, pp. 58-63.

61. Allen, F.R.; Ambikairajah, E.; Lovell, N.H. Classification of a known sequence of motions and postures from accelerometry data using adapted gaussian mixture models. Physiol. Meas. 2006, 27, 935-951. [CrossRef] [PubMed]

62. Preece, S.J.; Goulermas, J.Y.; Kenney, L.P.J. Acticity identification using body-mounted sensors-A review of classification techniques. Physiol. Meas. 2009, 1-33. [CrossRef] [PubMed]

63. Mendes, J.J.A.; Vieira, M.E.M.; Pires, M.B.; Stevan, S.L. Sensor fusion and smart sensor in sports and biomedical applications. Sensors 2016, 16, 1569. [CrossRef] [PubMed]

64. Bonacci, J.; Vicenzino, B.; Spratford, W.; Collins, P. Take your shoes off to reduce patellofemoral joint stress during running. Br. J. Sports Med. 2014, 48, 425-428. [CrossRef] [PubMed]

65. Snijders, A.H.; van de Warrenburg, B.P.; Giladi, N.; Bloem, B.R. Neurological gait disorders in elderly people: Clinical approach and classification. Lancet Neurol. 2007, 6, 63-74. [CrossRef]

66. Soh, S.E.; Morris, M.E.; McGinley, J.L. Determinants of health-related quality of life in parkinson's disease: A systematic review. Parkinsonism Relat. Disord. 2011, 17, 1-9. [CrossRef] [PubMed]

67. Sousa, A. Influence of long-term wearing of unstable shoes on compensatory control of posture: An electromyography-based analysis. Gait Posture 2014, 39, 98-104. [CrossRef] [PubMed]

68. Stolze, H.; Klebe, S.; Baecker, C.; Zechlin, C.; Friege, L.; Pohle, S.; Deuschl, G. Prevalence of gait disorders in hospitalized neurological patients. Mov. Disord. 2005, 20, 89-94. [CrossRef] [PubMed] 
69. Cheung, R.T.H.; Rainbow, M.J. Landing pattern and vertical loading rates during first attempt of barefoot running in habitual shod runners. Hum. Mov. Sci. 2014, 34, 120-127. [CrossRef] [PubMed]

70. Cronin, N.J. The effects of high heeled shoes on female gait: A review. J. Electromyogr. Kinesiol. 2014, 24, 258-263. [CrossRef] [PubMed]

71. Forghany, S.; Nester, C.J.; Richards, B.; Hatton, A.L.; Liu, A. Rollover footwear affects lower limb biomechanics during walking. Gait Posture 2014, 39, 205-212. [CrossRef] [PubMed]

72. Godinho, C.; Domingos, J.; Cunha, G.; Santos, A.T.; Fernandes, R.M.; Abreu, D.; Gonçalves, N.; Matthews, H.; Isaacs, T.; Duffen, J.; et al. A systematic review of the characteristics and validity of monitoring technologies to assess parkinson's disease. J. Neuroeng. Rehabil. 2016, 13, 24. [CrossRef] [PubMed]

73. Sánchez-Ferro, Á.; Elshehabi, M.; Godinho, C.; Salkovic, D.; Hobert, M.A.; Domingos, J.; van Uem, J.M.T.; Ferreira, J.J.; Maetzler, W. New methods for the assessment of parkinson's disease (2005 to 2015): A systematic review. Mov. Disord. 2016, 31, 1283-1292. [CrossRef] [PubMed]

74. Maetzler, W.; Klucken, J.; Horne, M. A clinical view on the development of technology-based tools in managing parkinson's disease. Mov. Disord. 2016, 31, 1263-1271. [CrossRef] [PubMed]

75. Klucken, J.; Friedl, K.E.; Eskofier, B.M.; Hausdorff, J.M. Guest editorial: Enabling technologies for parkinson's disease management. IEEE J. Biomed. Health Inform. 2015, 19, 1775-1776. [CrossRef] [PubMed]

76. Del Din, S.; Godfrey, A.; Mazzà, C.; Lord, S.; Rochester, L. Free-living monitoring of parkinson's disease: Lessons from the field. Mov. Disord. 2016, 31, 1293-1313. [CrossRef] [PubMed]

77. Caudron, S.; Guerraz, M.; Eusebio, A.; Gros, J.P.; Azulay, J.P.; Vaugoyeau, M. Evaluation of a visual biofeedback on the postural control in parkinson's disease. Clin. Neurophysiol. 2013, 44, 77-86. [CrossRef] [PubMed]

78. Horak, F.; King, L.; Mancini, M. Role of body-worn movement monitor technology for balance and gait rehabilitation. Am. Phys. Ther. Assoc. 2014, 95, 461-470. [CrossRef] [PubMed]

79. Schwenk, M.; Mohler, J.; Wendel, C.; D'Huyvetter, K.; Fain, M.; Taylor-Piliae, R.; Najafi, B. Wearable sensor-based in-home assessment of gait, balance, and physical activity for discrimination of frailty status: Baseline results of the arizona frailty cohort study. Gerontology 2015, 61, 10. [CrossRef] [PubMed]

80. Lee, S.I.; Park, E.; Huang, A.; Mortazavi, B.; Garst, J.H.; Jahanforouz, N.; Espinal, M.; Siero, T.; Pollack, S.; Afridi, M.; et al. Objectively quantifying walking ability in degenerative spinal disorder patients using sensor equipped smart shoes. Med. Eng. Phys. 2016, 38, 442-449. [CrossRef] [PubMed]

81. Lee, S.I.; Campion, A.; Huang, A.; Park, E.; Garst, J.H.; Jahanforouz, N.; Espinal, M.; Siero, T.; Pollack, S.; Afridi, M.; et al. Identifying predictors for postoperative clinical outcome in lumbar spinal stenosis patients using smart-shoe technology. J. Neuroeng. Rehabil. 2017, 14, 77. [CrossRef] [PubMed]

82. Perrier, A.; Vuillerme, N.; Luboz, V.; Bucki, M.; Cannard, F.; Diot, B.; Colin, D.; Rin, D.; Bourg, J.P.; Payan, Y. Smart diabetic socks: Embedded device for diabetic foot prevention. Innov. Res. BioMed. Eng. 2014, $32,5$. [CrossRef]

83. Ma, Y.; Amini, N.; Ghasemzadeh, H. Wearable sensors for gait pattern examination in glaucoma patients. Microprocess. Microsyst. 2016, 46, 67-74. [CrossRef]

84. Bertolucci, F.; Di Martino, S.; Orsucci, D.; Ienco, E.C.; Siciliano, G.; Rossi, B.; Mancuso, M.; Chisari, C. Robotic gait training improves motor skills and quality of life in hereditary spastic paraplegia. NeuroRehabilitation 2015, 36, 93-99. [PubMed]

85. Ossig, C.; Antonini, A.; Buhmann, C.; Classen, J.; Csoti, I.; Falkenburger, B.; Schwarz, M.; Winkler, J.; Storch, A. Wearable sensor-based objective assessment of motor symptoms in parkinson's disease. J. Neural Transm. (Vienna) 2016, 123, 57-64. [CrossRef] [PubMed]

86. Nonnekes, J.; Goselink, R.; Weerdesteyn, V.; Bloem, B.R. The retropulsion test: A good evaluation of postural instability in parkinson's disease? J. Parkinson Dis. 2015, 5, 43-47.

87. Bregou Bourgeois, A.; Mariani, B.; Aminian, K.; Zambelli, P.Y.; Newman, C.J. Spatio-temporal gait analysis in children with cerebral palsy using, foot-worn inertial sensors. Gait Posture 2014, 39, 436-442. [CrossRef] [PubMed]

88. Lees, A.J.; Hardy, J.; Revesz, T. Parkinson's disease. Lancet 2009, 373, 2055-2066. [CrossRef]

89. Goetz, C.G.; Tilley, B.C.; Shaftman, S.R.; Stebbins, G.T.; Fahn, S.; Martinez-Martin, P.; Poewe, W.; Sampaio, C.; Stern, M.B.; Dodel, R.; et al. Movement disorder society-sponsored revision of the unified parkinson's disease rating scale (mds-updrs): Scale presentation and clinimetric testing results. Mov. Disord. 2008, 23, 2129-2170. [CrossRef] [PubMed] 
90. van Uem, J.M.; Marinus, J.; Canning, C.; van Lummel, R.; Dodel, R.; Liepelt-Scarfone, I.; Berg, D.; Morris, M.E.; Maetzler, W. Health-related quality of life in patients with parkinson's disease-a systematic review based on the icf model. Neurosci. Biobehav. Rev. 2016, 61, 26-34. [CrossRef] [PubMed]

91. Maetzler, W.; Liepelt, I.; Berg, D. Progression of parkinson's disease in the clinical phase: Potential markers. Lancet. Neurol. 2009, 8, 1158-1171. [CrossRef]

92. Espay, A.J.; Bonato, P.; Nahab, F.B.; Maetzler, W.; Dean, J.M.; Klucken, J.; Eskofier, B.M.; Merola, A.; Horak, F.; Lang, A.E.; et al. Technology in parkinson's disease: Challenges and opportunities. Mov. Disord. 2016, 31, 1272-1282. [CrossRef] [PubMed]

93. Qiang, J.K.; Marras, C. Telemedicine in parkinson's disease: A patient perspective at a tertiary care centre. Parkinsonism Relat. Disord. 2015, 21, 525-528. [CrossRef] [PubMed]

94. Zampieri, C.; Salarian, A.; Carlson-Kuhta, P.; Nutt, J.G.; Horak, F.B. Assessing mobility at home in people with early parkinson's disease using an instrumented timed up and go test. Parkinsonism Relat. Disord. 2011, 17, 277-280. [CrossRef] [PubMed]

95. Salarian, A.; Horak, F.B.; Zampieri, C.; Carlson-Kuhta, P.; Nutt, J.G.; Aminian, K. Itug, a sensitive and reliable measure of mobility. IEEE Trans. Neural Syst. Rehabil. Eng. 2010, 18, 303-310. [CrossRef] [PubMed]

96. Forsaa, E.B.; Larsen, J.P.; Wentzel-Larsen, T.; Herlofson, K.; Alves, G. Predictors and course of health-related quality of life in parkinson's disease. Mov. Disord. 2008, 23, 1420-1427. [CrossRef] [PubMed]

97. Muro-de-la-Herran, A.; Garcia-Zapirain, B.; Mendez-Zorrilla, A. Gait analysis methods: An overview of wearable and non-wearable systems, highlighting clinical applications. Sensors 2014, 14, 3362-3394. [CrossRef] [PubMed]

(C) 2017 by the authors. Licensee MDPI, Basel, Switzerland. This article is an open access article distributed under the terms and conditions of the Creative Commons Attribution (CC BY) license (http:/ / creativecommons.org/licenses/by/4.0/). 